\title{
Robust Stability of Barrier-Based Model Predictive Control
}

\author{
Panagiotis Petsagkourakis, Student Member, IEEE, William P. Heath, Member, IEEE, Joaquin \\ Carrasco, Member, IEEE and Constantinos Theodoropoulos
}

\begin{abstract}
Conditions for robust input-output stability of barrier-based model predictive control of linear systems with linear and convex nonlinear (hard or soft) constraints are established through the construction of integral quadratic constraints (IQCs). The IQCs can be used to determine sufficient conditions for global closed-loop stability. In particular conditions for robust stability can be obtained in the presence of unstructured model uncertainty. IQCs with both static and dynamic multipliers are developed and appropriate convex searches for the multipliers are presented. The effectiveness of the robust stability analysis is demonstrated through an illustrative numerical example.
\end{abstract}

\section{INTRODUCTION}

Model predictive control (MPC) has been widely used to compute a sequence of control inputs online by optimizing an objective function with suitable constraints in a receding horizon manner. Nevertheless, it remains hard to guarantee robustness without introducing prohibitive complexity [1]. In this paper we obtain sufficient conditions for global robust stability for barrier-based MPC with linear or convex nonlinear input constraints where the plant is open-loop stable, through the use of IQCs, introduced in [2] to provide a unified framework for analyzing the robustness of Lur'e type systems. Classic robust control theory deals with stability and robustness under unstructured uncertainty, where IQCs are used to represent nonlinear and uncertain components [3]. Recent studies include robust stability analysis and perfomance [4] for LPV [5] and for distributed parameter systems [6]. IQCs can also be used to analyze the robust stability of anti-windup control systems with both unstructured uncertainty and nonlinearities [7]. Passivity, dissipativity and IQCs have been explored to analyze the input-output stability of MPC. IQC-based robust stability analysis of MPC has been performed in [8]. A robust output MPC design based on dissipativity for unstructured uncertainties has been proposed in [9]. More recently, IQCs in conjunction with dissipativity have been used for multi-model MPC [10] and later for LPV based MPC [11]. To overcome the extensive conservatism in MPC stability analysis, the existence of Zames-Falb (ZF) multipliers, originally proposed in [12], was shown in [13], for time-invariant linear constraints. In [14] necessary conditions have been provided for the existence of ZF multipliers for MIMO nonlinearities. Repeated nonlinearities have been treated through a special class of multipliers [15]. In [7] a unified framework for multiplier search by LMI optimization was formulated. Recently, a comprehensive analysis for slope-restricted nonlinearities in discrete time was proposed [16], [17]. Barrier-based MPC was proposed in [18] where stability of state feedback MPC can be established using gradient recentering; its implementation on an edible oil refining plant was reported in [19]. Fast implementations of MPC using barriers are reported in [20]. Recently, in a series of papers [21], [22] barrier-based MPC is developed to establish stability results for numerically efficient practical MPC implementations. Innovations of these works include weight-recentered barriers, analysis of relaxed barriers for soft constraints and stability results for anytime algorithms (where the number of Newton steps in the associated optimization algorithm is small). Additionally, barriers have been applied in the construction of barrier-based Lyapunov functions [23], [24] with state constraint satisfaction guarantees.

In this paper, we use recentered barriers for hard constraints and a relaxed recentered barrier for soft constraints. This complements the work in [22], [21], [25] which considers stability of state feedback MPC for soft input and state constraints. Here, stability analysis allows hard input constraints which may be time-variant or nonlinear. We show that the use of a barrier in the MPC formulation can be advantageous for computing the closed-loop stability, and we provide the appropriate analysis to verify this. In the case of time-invariant constraints, ZF multipliers can be used to reduce the inherent stability analysis conservatism. Hence, the search for such multipliers becomes crucial, and we construct static and dynamic multipliers, exploiting convex searches, for barrier-based MPC. Then input-to-output stability and robust analysis are performed, illustrating the advantages of barrier MPC. A significant benefit of this powerful methodology is that it can be naturally extended (as in [10]) towards nonlinear systems with unstructured uncertainty.

In section II the basic notations used in the paper are provided. In section III the formulation of barrier MPC is discussed and in section IV, some basic results are presented. Sections V and VI give our main results. The properties of barrier MPC are investigated, and the existence of static/ dynamic multipliers is shown. In section VII, a convex search methodology for efficiently computing multipliers is presented. In section VIII an illustrative numerical example is shown, while conclusions are given in IX.

\section{NOTATION}

Let $l^{m}$ be the space of all real-valued sequences. $\mathbf{R} \mathbf{H}_{\infty}$ is the set of rational matrix transfer function matrices without poles outside the unit circle. Let $x_{k} \in \mathbb{R}^{n_{x}}$ be the value of $x \in l^{n_{x}}$ at sample $k$. Let $A^{*}$ be the complex conjugate transpose of matrix $A$ and $G^{*}$ the $l_{2}$-adjoint operator of $G .\langle f, g\rangle$ is the inner product of real-valued sequences $f$ and $g$, defined as $\sum_{k=-\infty}^{\infty} f_{k}^{\top} g_{k}=\frac{1}{\pi} \int_{-\pi}^{\pi} \hat{f}\left(e^{j \omega}\right) \hat{g}\left(e^{j \omega}\right) d \omega, \hat{f}$ being the Fourier transform of $f . \sqrt{\langle f, f\rangle}$ is the $l_{2}$ norm $\|f\|_{2}$. The discrete convolution at time $i$ is $(f * g)_{i}=\sum_{k=-\infty}^{\infty} f_{k} g_{i-k}$. The size of signal $x$ is $n_{x} . I$ denotes the identity matrix. For a matrix $A \in$ $\mathbb{R}^{m \times n}$ with rank $r$ we define $A^{c} \in \mathbb{R}^{n-r \times n}$ such that $A^{c} A^{T}=0$, $A^{c} A^{c T}=I$, and $\bar{A} \in \mathbb{R}^{r \times n}$ such that 


$$
\bar{A}=\left\{\begin{array}{l}
\left(A^{c}\right)^{c}, \text { when } r<n \\
I \quad, \text { when } r=n .
\end{array}\right.
$$

Hence, $\bar{A} \bar{A}^{T}=I$ and the rows of $\bar{A}$ form an orthonormal basis of the space spanned by the rows of $A$. The set of all subgradients of a function $f$ at $x$ is called the subdifferential of $f$ at $x$ denoted by $\partial f=\partial f(x)$. Function $\phi: \mathbb{R}^{n_{x}} \rightarrow \mathbb{R}^{n_{y}}$ is defined for nonlinearity $\varphi: l_{2}^{n_{x}} \rightarrow l_{2}^{n_{y}}$ such that $(\varphi x)(t)=\phi(x(t))$.

\section{FORMULATION OF BARRIER MPC}

The controller is designed using the nominal LTI model (without any information for the disturbances):

$$
\begin{aligned}
& x_{k+1}=A x_{k}+B_{u} u_{k} \\
& y_{k}=C x_{k},
\end{aligned}
$$

where $x_{k} \in \mathbb{R}^{n_{x}}$ is the vector of states, $u_{k} \in \mathbb{R}^{n_{u}}$ the vector of manipulated variables, and $y_{k} \in \mathbb{R}^{n_{y}}$ the vector of measured output variables. Additionally, we assume that $A \in \mathbb{R}^{n_{x} \times n_{x}}$ is Schur stable. The formulation of barrier MPC is deduced from the nominal constrained problem, hence nominal MPC is presented first through the relevant control action:

$$
\begin{gathered}
U_{k}=\arg \min _{\tilde{u}_{k}} \frac{1}{2}\left[\sum_{i=1}^{N} \hat{x}_{k+i \mid k}^{T} Q \hat{x}_{k+i \mid k}+\sum_{i=0}^{N-1} \hat{u}_{k+i \mid k}^{T} R \hat{u}_{k+i \mid k}\right] \\
\text { s.t. } \hat{x}_{k \mid k}=x_{k} \\
\hat{x}_{k+i \mid k}=A \hat{x}_{k+i-1 \mid k}+B_{u} \hat{u}_{k+i-1 \mid k} \\
\hat{y}_{k+i-1 \mid k}=C \hat{x}_{k+i-1 \mid k} \\
\tilde{u}_{k}=\left[\begin{array}{lll}
\hat{u}_{k \mid k}^{T} & \ldots & \hat{u}_{k+N-1 \mid k}^{T}
\end{array}\right]^{T} \in \mathfrak{U}
\end{gathered}
$$

$N$ being the prediction horizon. The compact convex set $\mathfrak{U} \subseteq$ $\mathbb{R}^{n_{u}}$ represents the input constraints, $F_{i}: \mathbb{R}^{n \times n_{u}} \rightarrow \mathbb{R}$ :

$$
\mathfrak{U}:\left\{F_{i}(\tilde{u}) \leq W_{i} \mid i \in\{1, \ldots, n\}\right\},
$$

$F$ being a convex function. For the case of linear inequalities, the constraints can be written as $F(\tilde{u})=L \tilde{u} \leq W$, assuming $F_{i}(0)=0$ and $W_{i} \geq 0$. If we add the equality constraints (3b) to the objective function (3a) we obtain:

$$
\begin{aligned}
& U_{k}=\arg \min _{\tilde{u}} \frac{1}{2} \tilde{u}^{T} H \tilde{u}-\theta_{k}^{T} \tilde{u} \\
& \text { s.t. } \tilde{u} \in \mathfrak{U},
\end{aligned}
$$

where $H, R, Q$ are defined as in [26] and $\theta$ is a linear function of states $\left(\theta_{k}=-S x_{k}\right)$. The constrained problem is then transformed to an unconstrained problem as in [21], [18] using barrier functions and/or penalty functions (relaxed barrier).

Definition 1. Let $\mathscr{U}$ be an open (strictly) convex set which contains the origin, defined as $\mathscr{U}:=\cap \mathscr{U}_{i}$, where $\mathscr{U}_{i}=$ $\left\{\tilde{u}: F_{i}(\tilde{u}) \leq W_{i}\right\}$ in the case of hard constraints or $\mathscr{U}_{i}=$ $\mathbb{R}^{n_{U}}$ for soft constraints. Then $\mathscr{B}$ is the set of all twice continuous differentiable $\vartheta$-self-concordant (strictly) convex barrier functions over $\mathscr{U}, B: \mathscr{U} \rightarrow \mathbb{R}$ with $B(0)=0$ and $\nabla B(0)=0$.
In the literature there are two popular barrier functions utilized. The gradient recentered log-barrier [18]

$B(U)=\sum_{i} B_{i}=\sum_{i}\left(-\ln \left(W_{i}-F_{i}(U)\right)+\ln \left(W_{i}\right)-\frac{\nabla F_{i}(U)^{T}}{W_{i}-F_{i}(0)} U\right)$

and the weighted recentered log-barrier [21].

$$
B(U)=\sum_{i} B_{i}=\sum_{i}\left(1+w_{i}\right)\left(-\ln \left(W_{i}-F_{i}(U)\right)+\ln \left(W_{i}\right)\right)
$$

with $w_{i}>0$. In addition, a relaxed barrier function has been proposed [21] substituting the natural logarithm in (6) \& (7) with a quadratic function $\beta_{i}$ when $W_{i}-F_{i}(U) \leq \delta_{i}$ with $\delta_{i}>0$. The quadratic function is defined so that the properties from Definition 1 are maintained but the domain of the function $B_{i}$ is $\mathbb{R}^{n_{U}}$. The use of a barrier function allows the elimination of the inequality constraints.

$$
U_{k}=\phi\left(\theta_{k}\right)=\arg \min _{\tilde{u}} \frac{1}{2} \tilde{u}^{T} H \tilde{u}-\theta_{k}^{T} \tilde{u}+\mu B(\tilde{u}) .
$$

Let the function $\phi: \mathbb{R}^{n_{U}} \rightarrow \mathbb{R}^{n_{U}}$ be a family of MPCs, parametrised by $\mu$ (and $B$ ) generated by the nominal plant. The robustness of the system (2) with additional unstructured uncertainty and controlled by (8) is considered using static and dynamic multipliers for IQCs for the barrier-based MPC. For a general $\mathscr{U}$, static multipliers are utilized; if $\mathscr{U}$ is time-invariant (as is often the case) then the map $\phi$ is also time-invariant so dynamic multipliers can be explored.

\section{BACKGROUND}

\section{A. Properties of Nonlinear Functions}

A multi-valued map $\phi$ is sector-bounded in the sense that there exists some symmetric and positive definite $K \in \mathbb{R}^{n \times n}$, (or equivalently $\phi$ belongs to the sector $[0, K]$ ) such that

$$
\phi(\theta)^{T}\left(K^{-1} \phi(\theta)-\theta\right) \leq 0
$$

for all $\theta \in \mathbb{R}^{n}$ and it is additionally slope-restricted, if there is $S \in \mathbb{R}^{n \times n}, S>0$ such that for all $\theta_{x}, \theta_{y} \in \mathbb{R}^{n}$ and $\phi_{x}=\phi\left(\theta_{x}\right)$ :

$$
\left(\phi_{y}-\phi_{x}\right)^{T}\left(S^{-1}\left(\phi_{y}-\phi_{x}\right)-\left(\theta_{y}-\theta_{x}\right)\right) \leq 0 .
$$

Additionally, if $\phi(0)=0$ then a slope-restricted nonlinearity is also sector-bounded. Another property that we expoit here is cyclic monotonocity. A $n$-cyclic monotone increasing multivalued map $\phi$ is defined as follows:

Definition 2. ([27]) If $\phi$ is a n-cyclic monotone increasing map and $\phi_{i}=\phi\left(\theta_{i}\right)$ then $\forall n$

$$
\left\langle\theta_{0}-\theta_{1}, \phi_{0}\right\rangle+\left\langle\theta_{1}-\theta_{2}, \phi_{1}\right\rangle+\ldots+\left\langle\theta_{n}-\theta_{0}, \phi_{n}\right\rangle \geq 0 .
$$

The $n$-cyclic monotone is an extension of the monotone property. Namely for $n=1$, inequality (11) turns into the monotone increasing property and the existence of a convex gradient function is summarized in [27].

Theorem IV.1. [27] Let $\phi$ be a multi-valued mapping from $\mathbb{R}^{n} \rightarrow \mathbb{R}^{n}$. In order for a closed proper convex function $P$ on $\mathbb{R}^{n}$ such that $\phi(\theta) \subset \partial P$ for every $\theta$ to exist, it is necessary and sufficient that $\phi$ is cyclically monotone. 


\section{B. Integral Quadratic Constraints}

IQC's provide a way to conveniently represent associations between nonlinear or possibly unknown processes in frequency space, $\omega \in[-\pi, \pi]$ [3]. Two signals $w \in l_{2}^{n}$ and $v \in l_{2}^{n}$ (with Fourier tranforms $\hat{w}$ and $\hat{v}$, respectively) are said to satisfy the IQC defined by a multiplier $\Pi$, which is measurable, bounded and Hermitian, if

$$
\int_{-\pi}^{+\pi}\left[\begin{array}{c}
\hat{w}\left(e^{j \omega}\right) \\
\hat{v}\left(e^{j \omega}\right)
\end{array}\right]^{*} \Pi\left(e^{j \omega}\right)\left[\begin{array}{c}
\hat{w}\left(e^{j \omega}\right) \\
\hat{v}\left(e^{j \omega}\right)
\end{array}\right] d \omega \geq 0 .
$$

The classic stability theorem presented in [3], assumes that the interconnection between the system transfer function $G$ and the augmented nonlinearity $\Delta$ is well-posed. In addition the feedback interconnection between $G$ and $\Delta$ is stable if there exists $\varepsilon>0$ such that

$$
\left[\begin{array}{c}
G\left(e^{j \omega}\right) \\
I
\end{array}\right]^{*} \Pi\left(e^{j \omega}\right)\left[\begin{array}{c}
G\left(e^{j \omega}\right) \\
I
\end{array}\right] \leq-\varepsilon I .
$$

\section{Zames-Falb Multipliers}

Definition 3. ([14]) The class of discrete-time rational ZamesFalb multipliers $\mathscr{M}$ contains all MIMO rational transfer functions $M_{Z F} \in \mathbf{R L}_{\infty}^{n \times n}$ such that $M_{Z F}(z)=H_{s}-H_{Z F}(z)$. Let $H_{Z F}$ denote an $n \times n$ symmetric matrix-valued function with entries in $l_{1}$, and also let $H_{s}$ be an $n \times n$ real symmetric matrix with $M_{Z F}$ being symmetric doubly hyper-dominant [28]:

$$
H_{s_{i i}} \geq \sum_{j, j \neq i}\left|H_{s_{i j}}\right|+\sum_{j}\left\|H_{Z F_{i j}}\right\|_{1}
$$

with $H_{Z F_{i j}}$ and $H_{s_{i j}}$ being elements of matrices $H_{Z F}$ and $H_{s}$ respectively. Additionally, the subclass $\mathscr{M}_{+} \subset \mathscr{M}$ requires the following:

$$
H_{s_{i j}} \leq 0, H_{Z F_{i j}} \geq 0
$$

\section{Properties for Barrier MPC}

In this section the properties related to barrier MPC are explored in the context of IQCs. The next two lemmas are required to show that $\phi: \theta_{k} \mapsto U_{k}(8)$ is slope-restricted, sectorbounded and cyclic monotone.

\section{Lemma V.1.}

1) If $B \in \mathscr{B}$, then $\nabla B$ is monotone increasing and there exists $m \geq 0$ such that $\nabla^{2} B \geq m I$ and $U^{T} \nabla B-m U^{T} U \geq 0$.

2) If $B$ is also strongly convex then we can find $m>0$.

Proof:

1) This is trivial when $m=0$ through convexity.

2) If $B$ is strongly convex then we can find $m>0$ such that $\nabla^{2} B-m I \geq 0$. Define $\bar{B}=B-\frac{1}{2} m U^{T} U$. Then $\bar{B}$ is convex and the result follows since $U^{T}(\nabla B-m U)=U^{T} \nabla \bar{B} \geq 0$.

Lemma V.2. If $\mathscr{U}$ is an open (strictly) convex set which contains the origin, given by Definition 1 and is also compact, $B \in \mathscr{B}$ and the constraints $\mathfrak{U}$ are hard or $B$ is relaxed by a quadratic function $\beta_{i}[21]$, then the recentered barrier is strongly convex with $m>0$.
Proof: For the set described by Definition 1, there is a finite positive $\delta_{e}$ such that $b_{i}-F_{i}(U)=b_{i}-L_{i} U \leq \delta_{e}$. For a given $U \in \mathscr{U}$, the following holds:

$$
\nabla^{2} B_{i}=\frac{L_{i}^{T} L_{i}}{\left(b_{i}-L_{i} U\right)^{2}} \geq \frac{L_{i}^{T} L_{i}}{\delta_{e}^{2}} \geq 0 .
$$

It is trivial to show that there exits a $B_{j}$ such that $\nabla^{2} B_{i}+$ $\nabla^{2} B_{j}>0$.

In the rest of the paper the matrix $\tilde{H}$ is defined as:

$$
\tilde{H}=H+\mu m I .
$$

Theorem V.3. The nonlinearity $\phi: \mathbb{R}^{n_{U}} \rightarrow \mathbb{R}^{n_{U}}$ (8) belongs to the sector $\left[0, \tilde{H}^{-1}\right]$, with $m \geq 0$ from lemma V.1 and V.2, $B \in \mathscr{B}$ and $\mathscr{U}$ is a convex set, if $\phi(0)=0$.

Proof: Using the KKT conditions of (8) and with $\phi=$ $\phi(\theta) \in \mathscr{U}$ we have:

$$
H \phi-\theta+\mu \nabla B(\phi)=0 .
$$

Since $\mathscr{U}$ is convex, multiplying (18) by $U^{T}$, using lemma V.1 we get:

$$
\phi^{T}(H+\mu m I) \phi-\phi^{T} \theta \leq 0 .
$$

Theorem V.4. The nonlinearity $\phi: \mathbb{R}^{n_{U}} \rightarrow \mathbb{R}^{n_{U}}$ (8) is additionally slope-restricted on $\left[0, \tilde{H}^{-1}\right]$ with $m \geq 0, B \in \mathscr{B}$ and $\mathscr{U}$ is given by Definition 1.

Proof: Using the KKT conditions of (8) we have the following for $\phi_{x}=\phi\left(\theta_{x}\right) \in \mathscr{U}$ and $\phi_{y}=\phi\left(\theta_{y}\right) \in \mathscr{U}$

$$
\begin{aligned}
& H \phi_{x}-\theta_{x}+\mu \nabla B\left(\phi_{x}\right)=0 \\
& H \phi_{y}-\theta_{y}+\mu \nabla B\left(\phi_{y}\right)=0 .
\end{aligned}
$$

Subtract (20b) from (20a) and multiply by $\left(\phi_{y}-\phi_{x}\right)^{T}$ to get:

$$
\begin{aligned}
& \left(\phi_{x}-\phi_{y}\right)^{T}\left(H\left(\phi_{x}-\phi_{y}\right)-\left(\theta_{x}-\theta_{y}\right)\right)+ \\
& +\left(\phi_{x}-\phi_{y}\right)^{T}\left(\mu \nabla B\left(\phi_{x}\right)-\mu \nabla B\left(\phi_{y}\right)\right)=0 .
\end{aligned}
$$

Applying Lemma V.1\&V.2:

$$
\begin{aligned}
& \left(\phi_{x}-\phi_{y}\right)^{T}\left(H\left(\phi_{x}-\phi_{y}\right)-\left(\theta_{x}-\theta_{y}\right)\right)= \\
& -\left(\phi_{x}-\phi_{y}\right)^{T}\left(\mu \nabla B\left(\phi_{x}\right)-\mu \nabla B\left(\phi_{y}\right)\right) \leq \mu m\left\|\phi_{x}-\phi_{y}\right\|^{2} .
\end{aligned}
$$

Then $\left(\phi_{x}-\phi_{y}\right)^{T}\left((H+\mu m I)\left(\phi_{x}-\phi_{y}\right)-\left(\theta_{x}-\theta_{y}\right)\right) \leq 0$. Therefore, $\phi$ is slope-restricted on $\left[0, \tilde{H}^{-1}\right]$.

It should be mentioned that for the sector bounded result there is no requirement for $\mathscr{U}$ being time-invariant.

Remark 1. This result shows that inclusion of a barrier can change the maximum slope of the input-output map of the controller, which widens the stability region of the closed-loop system. Here, $m$ depends only on the set of constraints and not on the design parameter $\mu$. In the numerical examples below, it will be shown that such a formulation can reduce conservatism significantly in comparison to [8].

We utilize a simple example to illustrate the effect of barrierbased MPC on the maximum slope. The nonlinearity is given by $U=\left(\arg \min _{u} 0.25 u^{2}-\theta u+\mu(-\ln (1-u)-\ln (2+u)-\right.$ $0.5 u$ ). Fig. 1 depicts the solution for different values of $\mu$. 


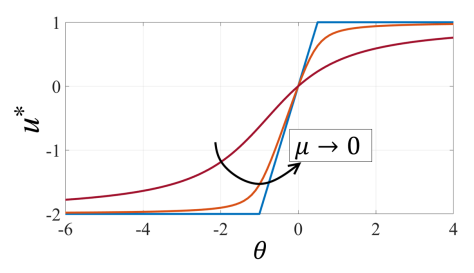

Fig. 1. Simple example showing that if a nonlinearity is parametrized by $\mu$ then its slope varies with $\mu$.

In this case the value of $m$ (and the maximum slope) can be computed analytically (See Appendix A), namely $m=0.889$ and Slope $_{\max }=(0.5+8 / 9 \mu)^{-1}$. Fig. 1 shows that the maximum slope decreases as $\mu$ increases. If $m$ is not computed (and is e.g. assumed to be $m=0$ ) then the maximum slope will be overestimated compared to the actual value of the slope, hence increasing the conservatism of the stability analysis.

Remark 2. The parameter $m$ cannot always be calculated analytically. The search for $m$ may not be trivial and a signomial programming problem [29] is formulated such that the minimum eigenvalue of $\nabla^{2} B$ is computed through deterministic methods. In Appendix $A$ it is shown that for the case of box constraints, $m$ can be calculated analytically. For general stage constraints the problem can be decomposed to smaller problems, solved using deterministic global optimization.

The barrier MPC is cyclic monotone when the convex set $\mathscr{U}$ is time-invariant.

Theorem V.5. When $B \in \mathscr{B}$ and in addition $\mathscr{U}$ is a timeinvariant convex set, then the nonlinearity $\phi: \mathbb{R}^{n_{U}} \rightarrow \mathbb{R}^{n_{U}}$ (8) is $n$-cyclic monotone.

Proof: For the barrier MPC the following hold:

$$
\begin{aligned}
& \sum_{k=0}^{n} \phi_{k}^{T}\left(\theta_{k}-\theta_{k+1}\right)= \\
& \sum_{k=0}^{n} \phi_{k}^{T}\left(H\left(\phi_{k}-\phi_{k+1}\right)+\mu\left(\nabla B\left(\phi_{k}\right)-\nabla B\left(\phi_{k+1}\right)\right)\right)=
\end{aligned}
$$$$
\left[\begin{array}{llll}
\phi_{0} & \phi_{1} & \ldots & \phi_{n}
\end{array}\right] A_{c}\left[\begin{array}{llll}
\phi_{0} & \phi_{1} & \ldots & \phi_{n}
\end{array}\right]^{T}+
$$$$
+\mu \sum_{k=0}^{n} \nabla B\left(\phi_{k+1}\right)^{T}\left(\phi_{k+1}-\phi_{k}\right),
$$

where $\phi_{n+1}=\phi_{0}, \theta_{n+1}=\theta_{0}$ and

$$
A_{c}=\frac{1}{2}\left[\begin{array}{ccccccc}
2 H & -H & 0 & \cdots & & 0 & -H \\
-H & 2 H & -H & & & & 0 \\
0 & -H & 2 H & & & & \\
\vdots & & & \ddots & & & \vdots \\
0 & & & & 2 H & -H & 0 \\
-H & 0 & & \cdots & 0 & -H & 2 H
\end{array}\right] .
$$

The matrix $A_{c}$ is always symmetric diagonally dominant with positive diagonal elements, thus it is positive semidefinite according to Gershgorin circle theorem [30], so:

$$
\left[\begin{array}{llll}
\phi_{0} & \phi_{1} & \ldots & \phi_{n}
\end{array}\right] A_{c}\left[\begin{array}{llll}
\phi_{0} & \phi_{1} & \ldots & \phi_{n}
\end{array}\right]^{T} \geq 0 .
$$

Since $B$ is convex its gradient is cyclic monotone:

$$
\begin{aligned}
& \nabla B\left(\phi_{0}\right)^{T}\left(\phi_{0}-\phi_{n}\right)+\nabla B\left(\phi_{n}\right)^{T}\left(\phi_{n}-\phi_{n-1}\right)+\ldots \\
& +\nabla B^{T}\left(\phi_{1}\right)\left(\phi_{1}-\phi_{0}\right) \geq 0 .
\end{aligned}
$$

Thus the non-linearity is cyclic monotone.

\section{MULTIPLIERS FOR BARRIER MPC}

IQCs for the barrier MPC will be derived in this section using the results from Section V.

\section{A. Static Multipliers}

Corollary 1. For $\mathscr{U}$ being a convex set and $B \in \mathscr{B}$, the nonlinearity $\varphi: l_{2}^{N_{U}} \rightarrow l_{2}^{N_{U}}$ (8), $\forall \theta \in l_{2}^{N_{U}}$ satisfies IQC with the following mulitiplier

$$
\left[\begin{array}{cc}
0 & I \\
I & -2 \tilde{H}
\end{array}\right] .
$$

Proof: Immediate from Theorem V.3.

Remark 3. Theorem V.3 introduces an IQC for the barrier $M P C$ where the constraints are generally convex (as long as the optimization problem is feasible). This case will be referred to as general. Nevertheless, by tightening the class of constraints, less conservative results can be computed.

\section{B. Dynamic Multipliers}

Time-invariant constraints can be used next to derive dynamic multipliers for less conservative stability analysis. We prove the existence of ZF multipliers for the case of timeinvariant convex constraints in Lemma VI.1, and of less conservative multipliers for box and staged constraints. According to [13], [14], the existence of ZF multipliers additionally requires the line integral $\int_{A}^{B} \phi(x)^{T} d x$ to be independent of the path. This property is equivalent to $\phi$ being the gradient of some convex function. When, however, the nonlinearity is not explicitly given, the above properties are difficult to be computed. Here we propose to use cyclic monotonicity in order to prove the existence of the convex function. From Theorem IV.1, the conditions in [13], [14] can be substituted by the condition of $\phi$ being cyclically monotone. Additionally, $\phi$ is bounded by $c$ if $\|\phi(\theta)\| \leq c\|\theta\| \forall \theta \in \mathbb{R}^{n}$.

Lemma VI.1. Let $\phi: \mathbb{R}^{n} \rightarrow \mathbb{R}^{n}$ be bounded and cyclic monotone increasing. Then for any $\theta \in l_{2}^{n}$ we have

$$
\sum_{t=-\infty}^{\infty} \theta_{t+\tau}^{T} \phi\left(\theta_{t}\right) \leq \sum_{t=-\infty}^{\infty} \theta_{t}^{T} \phi\left(\theta_{t}\right)
$$

and if $\phi$ is odd then

$$
\left|\sum_{t=-\infty}^{\infty} \theta_{t+\tau}^{T} \phi\left(\theta_{t}\right)\right| \leq \sum_{t=-\infty}^{\infty} \theta_{t}^{T} \phi\left(\theta_{t}\right) .
$$

Proof: From Theorem IV.1, it is necessary and sufficient that the mapping is cyclically monotone, for a closed proper convex function $P \in \mathbb{R}^{n}$ that satisfies $\nabla P(\theta)=\phi(\theta), P(0)=0$. Since $\phi$ is cyclically monotone non-decreasing,

$$
P(b)-P(a) \leq(b-a)^{T} \phi(b) .
$$


Also $\phi(\theta) \in l_{2}^{n}$ and $P(\theta) \in l_{1}$ for all $\theta \in l_{2}^{n}$. Let $b=\theta_{t}$ and $a=$ $\theta_{t+\tau}$. Summing equation (29) with respect to $t$ over $(-\infty,+\infty)$, equation (27) follows. Additionally, if $\phi(\theta)$ is odd, then $P(\theta)$ is an even function. For $a=-\theta_{t+\tau}$ and $b=\theta_{t}$

$$
-\sum_{t=-\infty}^{\infty} \theta_{t+\tau}^{T} \phi\left(\theta_{t}\right) \leq \sum_{t=-\infty}^{\infty} \theta_{t}^{T} \phi\left(\theta_{t}\right)
$$

and equation (28) follows due to (27).

Next, the theorem for the existence of ZF mutipliers is given:

Theorem VI.2. Let the nonlinearity $\varphi: l_{2}^{n_{U}} \rightarrow l_{2}^{n_{U}}$ be bounded, $n$-cyclic monotone increasing and slope-restricted, with slope $\tilde{H}$. Let the SISO multiplier be $M \in \mathscr{M}_{+}$(or $M \in \mathscr{M}$ and $\phi$ is additionally odd), then $M_{Z F}=M I$, and $\forall \theta \in l_{2}^{n_{U}}, \phi$ satisfies IQCs with the following multipliers:

$$
\Pi(z)=\left[\begin{array}{cc}
0 & M_{Z F}^{*}(z) \\
M_{Z F}(z) & -\tilde{H} M_{Z F}(z)-M_{Z F}^{*}(z) \tilde{H}
\end{array}\right] .
$$

Proof: Use Lemma VI.1 and [13].

Remark 4. If there is no special structure, e.g. repeated nonlinearities, then SISO multipliers should be used in the form of $M_{Z F}=M I$ with $M \in \mathscr{M}_{S I S O}$. $\mathscr{M}_{\text {SISO }}\left(\right.$ or $\left._{M_{S I S O}}\right)$ contains all SISO rational transfer functions $M_{Z F} \in \mathbf{R L}_{\infty}$ that maintain the properties of Definition 3.

Corollary 2. The nonlinearity $\phi: \mathbb{R}^{n_{U}} \rightarrow \mathbb{R}^{n_{U}}$ (8) satisfies IQC with multiplier $\Pi(z)$ (equation (31)), $\forall \theta \in l_{2}^{N_{U}}$ with $\mathscr{U}$ a time-invariant convex set and $B \in \mathscr{B}$.

Proof:

(a) The proof is almost identical with the one from [13]. (b) It follows immediately from Theorem V.5 that $\phi$ is cyclic monotone. (c) It follows immediately from Theorem V.4 that $\phi$ is slope-restricted on $\left[0, \tilde{H}^{-1}\right]$.

The above results can be exploited to provide ZF multipliers when (possibly relaxed) barrier MPC is utilized.

\section{Multipliers for Box/Staged Constraints}

The conservatism can be reduced even further when a special structure of constraints (linear set) is used and more general multipliers than SISO ZF can be utilized. The following analysis generalizes the results from [31], extending them to the case of (possibly relaxed) recentered Barrier MPC. The key idea is to represent the nonlinear function $\phi$ as an equivalent feedback structure. This structure is then formulated into a nonlinear program $\psi$ together with a linear feedback term, which can be separated into several smaller parallel nonlinear programs $v_{i}$. Multipliers can then be associated with each $v_{i}$. In our results, we show that there is a class of MIMO ZF multipliers $M_{Z F}$ for a special structure of staged and box constraints. Let $\psi: \mathbb{R}^{n_{U}} \rightarrow \mathbb{R}^{n_{U}}$ be the convex program:

$$
U=\psi\left(\theta^{\prime}\right)=\arg \min _{u} \frac{1}{2} u^{T} u-u^{T} \theta^{\prime}+\mu B(u)
$$

with $B \in \mathscr{B}$.

Lemma VI.3. If $\theta^{\prime}$ is equal to $\theta+(I-\tilde{H}) \phi(\theta)$, then $U=$ $\phi(\theta)$ and $U=\psi\left(\theta^{\prime}\right)$ are equivalent.

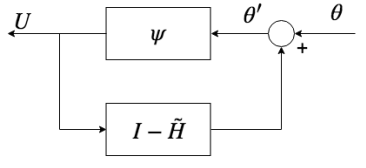

Fig. 2. Equivalent feedback structure

Proof: Substituting $\theta^{\prime}=\theta+(I-\tilde{H}) \phi(\theta)$ in the KKT conditions of (32), the result follows immediately.

This equivalent feedback structure is depicted in Fig. 2. A direct consequence of lemma VI.3 is the following:

$$
\left[\begin{array}{l}
\theta^{\prime} \\
U
\end{array}\right]=\left[\begin{array}{cc}
I & I-\tilde{H} \\
0 & I
\end{array}\right]\left[\begin{array}{l}
\theta^{\prime} \\
U
\end{array}\right]
$$

The rest of the analysis is based on the fact that $U=\psi\left(\theta^{\prime}\right)$ can be written as many parallel convex programs with $U=$ $\sum_{i=0}^{N_{L-1}} u_{i}$. To do so, special structures of the constraints are considered such as limitations between adjacent actuators' movement (so-called staged constraints) [31] as well as box constraints. Both cases can be written as:

$$
L=\left[\begin{array}{lll}
L_{0}^{T} & \ldots & L_{N_{L}}^{T}
\end{array}\right]^{T}=\operatorname{diag}\left(\tilde{L}_{0}, \ldots, \tilde{L}_{N_{L}-1}\right)
$$

with $L_{i}$ having the following property:

$$
L_{i} L_{j}^{T}=0
$$

for $i \neq j$. Hence, $U=\psi\left(\theta^{\prime}\right)$ can now be written as a set of parallel convex programs $\left(u_{i}\right)$ for the case of staged and box constraints, $U=\sum_{i} u_{i}$ with $u_{i}$ being a convex program $\psi_{i}\left(\theta^{\prime}\right)$.

Lemma VI.4. The nonlinear convex program $U=\psi\left(\theta^{\prime}\right)$, given by (32), can equivalently be transformed to $U=\sum_{i} u_{i}$, with $u_{i}$ being parallel convex programs and $\beta_{i} j$ a quadratic function of the $i^{\text {th }}$ convex program (see Lemma V.2):

$$
\begin{aligned}
& u_{i}=\arg \min _{u} \frac{1}{2} u^{T} u-u^{T} \theta^{\prime}+\mu\left(\sum_{j=1}^{N_{0}} \bar{B}_{i j}(u)+\ln \left(b_{i j}\right)-\frac{L_{i j}^{T}}{b_{i j}} u\right) \\
& \text { s.t. } L_{i}^{c} u=0 \\
& \bar{B}_{i j}(u)=\left\{\begin{array}{l}
-\ln \left(b_{i j}-L_{i j} u\right) \text { for }-L_{i j} U+b_{i j} \geq \delta \\
\beta_{i j}(u) \text { elsewhere }
\end{array}\right.
\end{aligned}
$$

Proof: See Appendix B

Now, let $\bar{L}_{i}$ be an orthonormal basis of the space spanned by the rows of $L_{i}$ and $v_{i}(p)$ be the convex program

$$
v_{i}(p)=\arg \min _{q} \frac{1}{2} q^{T} q-q^{T} p+\mu\left(\sum_{j=1}^{N_{0}} \bar{B}_{i j}\left(\bar{L}_{i j}^{T} q\right)-\frac{\bar{L}_{i j} L_{i j}^{T}}{b_{i j}} q\right) .
$$

Each $v_{i}$ is bounded, n-cyclic monotone and slope restricted with slope $I$. Theorem VI.2 can, therefore, be applied:

Lemma VI.5. Let $M_{Z F_{i}} \in \mathscr{M}_{+}$be a SISO rational strictly proper transfer function. Then $\forall p \in l_{2}^{n_{p}}, v_{i}$ satisfies IQC with the following multiplier:

$$
\Pi_{v i}(z)=\left[\begin{array}{cc}
0 & M_{Z F i}^{*} \\
M_{Z F i} & -M_{Z F i}-M_{Z F i}^{*}
\end{array}\right] .
$$


Proof: $v_{i}$ is bounded, $n$-cyclic monotone and slope restricted with slope $(I)$. Theorem VI.2 provides the result.

The next lemma shows that each $u_{i}$ can be written as a function of $v_{i}$.

Lemma VI.6. Each $u_{i}$ can equivalently be written as

$$
u_{i}=\bar{L}_{i}^{T} v_{i}\left(\bar{L}_{i} \theta^{\prime}\right) .
$$

Proof: See Appendix C

Consequently an IQC for the nonlinear system $\psi\left(\theta^{\prime}\right)$ with the following multiplier can be formulated:

$$
\Pi_{\psi}(z)=\sum_{i=0}^{N_{L}-1}\left[\begin{array}{cc}
\bar{L}_{i} & 0 \\
0 & \bar{L}_{i}
\end{array}\right]^{T} \Pi_{v_{i}}(z)\left[\begin{array}{cc}
\bar{L}_{i} & 0 \\
0 & \bar{L}_{i}
\end{array}\right]
$$

Theorem VI.7. Let $\varphi: l_{2}^{n_{U}} \rightarrow l_{2}^{n_{U}}$ be bound, n-cyclic monotone increasing and slope-restricted, with slope $\tilde{H}$ under box or staged constraints. Let the multiplier $M_{Z F_{i}} \in \mathscr{M}_{+}$be a SISO rational strictly proper transfer function,

$$
M_{Z F}(z)=\operatorname{diag}\left(M_{Z F_{0}} I, \ldots, M_{Z F_{\left(N_{L}-1\right)}} I\right)
$$

then $\forall \theta \in l_{2}^{n_{U}}$, $\phi$ satisfies IQC with the following multiplier:

$$
\begin{aligned}
& \Pi(z)=\left[\begin{array}{cc}
I & I-\tilde{H} \\
0 & I
\end{array}\right]^{T} \Pi_{\psi}(z)\left[\begin{array}{cc}
I & I-\tilde{H} \\
0 & I
\end{array}\right]= \\
& {\left[\begin{array}{cc}
0 & M_{Z F}^{*} \\
M_{Z F} & -\tilde{H} M_{Z F}-M_{Z F}^{*} \tilde{H}
\end{array}\right]}
\end{aligned}
$$

Proof: See Appendix D

This theorem can be further extended for an even tighter class of box constraints where symmetric bounds are employed. That is, full-block doubly hyper-dominant multipliers [28] can be applied as it can be proven that $\psi$ can be written as a linear transformation of repeated nonlinearities.

\section{CONVex Search fOR Multipliers}

In this section the convex search applied to the stability analysis is presented. The results in this work allow the use of static multipliers for a wide class of constraints or dynamic multipliers for a tighter class of constraints. We revisit [16], [17] and expand the results from [17], in order to incorporate a larger class of problems, where the slopes are given by a fullblock matrix. Dynamic multipliers may be non-causal and a factorization is required since they do not have a state-space representation. For the $i^{\text {th }}$ IQC its multiplier can be written as:

$$
\Pi_{i}(z)=\Psi^{*}(z) K_{i} \Psi(z)=\Psi^{*}(z)\left[\begin{array}{cc}
M_{i}^{11} & M_{i}^{12} \\
M_{i}^{12 T} & M_{i}^{22}
\end{array}\right] \Psi(z)
$$

For $N$ IQCs then we can then write:

$$
\Pi(z)=\Psi^{*}(z) K \Psi(z),
$$

with $K$ being defined in Appendix F. In this work, finite impulse response (FIR) type multipliers (43) are used. Their use can be justified using the phase equivalence argument [32].

$$
M_{Z F}=\sum_{j=-N_{Z F_{-}}}^{N_{Z F_{+}}} R_{j}\left(1-z^{j}\right) .
$$

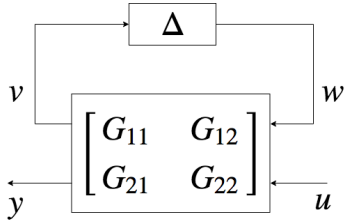

Fig. 3. Linear system under uncertainty

Using $N_{Z F}=\max \left(N_{Z F_{+}}, N_{Z F_{-}}\right), \Psi$ can be defined as:

$$
\begin{aligned}
& \Psi_{11}=\left[\begin{array}{llll}
I & \left(1-z^{-1}\right) I & \ldots & \left(1-z^{-N_{Z F}}\right) I
\end{array}\right]^{T} \\
& \Psi=\operatorname{diag}\left(\Psi_{11}, \Psi_{11}\right) .
\end{aligned}
$$

The slope-restricted nonlinearities described in this work satisfy IQC multipliers as in (41). The resulting $M_{\phi}^{11}, M_{\phi}^{11}$ and $M_{\phi}^{11}$ are shown in Appendix E. Therefore, the dynamic system $G_{\Psi}(z)$ has non-singular state-space representation and the LMI conditions can be constructed with state space realization of $\left(A_{\psi}, B_{\psi}, C_{\psi}, D_{\psi}\right)$ and $G_{\psi}=C_{\psi}\left(z I-A_{\psi}\right)^{-1} B_{\psi}+D_{\psi}$ :

$$
G_{\Psi}(z)=\Psi(z)\left[\begin{array}{c}
G(z) \\
I
\end{array}\right]
$$

Through the KYP lemma [33], inequality (13) can be transformed into the following LMI optimization:

$$
\begin{aligned}
& \min _{\lambda, K} \lambda \\
& \text { s.t. }\left[\begin{array}{cc}
A_{\Psi}^{T} P A_{\Psi}-P & A_{\Psi}^{T} P B_{\Psi} \\
B_{\Psi}^{T} P A_{\Psi} & B_{\Psi}^{T} P B_{\Psi}
\end{array}\right]+ \\
& {\left[\begin{array}{cc}
C & D \Psi \\
0 & I
\end{array}\right]^{T} K\left[\begin{array}{cc}
C & D \Psi \\
0 & I
\end{array}\right] \leq-\lambda I,}
\end{aligned}
$$

and additional constraints for the multipliers

The additional constraints depend of the class of the multipliers (if $M \in \mathscr{M}_{+}$or $\mathscr{M}$ ). For static multipliers, $R_{j}$ can be set equal to zero for $j \neq 0$. For diagonal multipliers(for example when asymmetric box or stage constraints are applied, $C-Z F)$, then $R_{j} \geq 0$ for $j \neq 0$ and $R_{0}>0$. The condition of doubly hyper-dominance can be similarly expressed.

\section{ROBUSTNESS OF BARRIER MPC}

Here the robustness of the barrier MPC in terms of input-tooutput stability is considered through an illustrative numerical example. If the transfer function of the open-loop LTI plant in (2) is $G_{22}$, the system under the unstructured uncertainty $\Delta: l^{n_{v}} \rightarrow l^{n_{w}}$, which satisfies an IQC, is given by Fig. 3. Then, the barrier MPC $(\phi)$ can be included in the analysis as in [7] with $\Delta=\left[\begin{array}{ll}\Delta_{1} & \\ & \phi\end{array}\right]$.

\section{A. Numerical Example}

A plant is described as

$\frac{y(z)}{u(z)}=$

$\frac{z^{4}+1.21 z^{3}+0.0238 z^{2}+\left(b_{0}-4.7610^{-6}\right) z+1.19\left(b_{0}-810^{-8}\right)}{z^{5}-0.69 z^{4}-0.25 z^{3}-4.6610^{-3} z^{2}+9.9 \times 10^{-7} z+1.9 \times 10^{-3}}$, 
where $y$ and $u$ are the output and input of the plant and $b_{0}>0$ is an uncertain parameter with maximum value $b$. In Figure 5 (a) the Nyquist diagram for different $b_{0}$ is given. The behaviour of the plant is standard when model-mismatch is present [34]. It is assumed that the plant in (47) is a reduced and separated nominal plant under unstructured uncertainty $\Delta_{1}: l \rightarrow l$ which is norm bounded as $\left\|\Delta_{1}\right\| \leq b$. The nominal dynamic system is given by the following equation:

$$
y(z)=\frac{z+1.19}{z^{2}-0.71 z-0.233} u(z) .
$$

For this example the LTI plant has eigenvalues 0.9542, 0.2442 and zero equal to -1.19 . As a result, the system is non-minimum phase. In addition to the nominal plant, the state observer is given by

$$
\hat{x}(t)=J_{u}(z) u(t)+J_{y}(z) y(t) .
$$

Here a steady-state Kalman filter is used with $J_{u}(z)=(z I-$ $A+A L C)^{-1} B$ and $J_{y}=(z I-A+A L C)^{-1} A L$. For the numerical example the observer gain $L$ was calculated via the discrete algebraic Riccati equation with weighting matrices set equal to the identity matrix. The linear part of Fig. 4 can be transformed

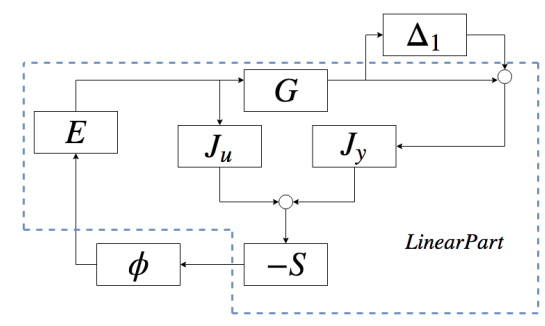

Fig. 4. Control Scheme

into the augmented linear system $M_{S}$

$$
M_{s}(z)=\left[\begin{array}{cc}
\sqrt{b} I & \\
& -S
\end{array}\right]\left[\begin{array}{cc}
0 & G \\
J_{y} & J_{u}+J_{y} G
\end{array}\right]\left[\begin{array}{ccc}
\sqrt{b} & I & \\
& E
\end{array}\right]
$$

with $I$ and 0 the identity and zero matrix, respectively. Only the control action is applied and hence $E=\left[\begin{array}{llll}I & 0 & \cdots & 0\end{array}\right]$. Additionally, the scaled uncertainty is defined as $\hat{\Delta}_{1}=b \Delta_{1}$ with $\left\|\hat{\Delta}_{1}\right\| \leq 1$ and $\phi$ the input-output map of the barrier MPC. The control action is given by (8), where $\theta=-S x$ and $B$ the gradient re-centered barrier function. As a result two IQCs can be written: one for the controller and one for the given unstructured uncertainty. For $\hat{\Delta}_{1}$ we have $\Pi_{\Delta_{1}}=\left[\begin{array}{cc}I & 0 \\ 0 & -I\end{array}\right]$. For the controller different multipliers are utilized depending on the case. All the algorithms presented in this work have been implemented for $\mu=0.8$ and sufficiently large $N_{Z F}$. The constraints added to the manipulated variables are $-0.5 \leq u_{k} \leq$ 1.0. The control and prediction horizon are both set equal to 2 and $Q=I$. Two problems are investigated: Task 1. A positive gain $\kappa>1$ is applied to the output of the dynamical system for $b=0$ and the goal is to compute the maximum stable gain. Task 2 is aimed at finding the smallest positive parameter $r$ of the objective function for $b=0.25$ and also the largest positive $b$ for $r=0.001$ so that the system is guaranteed stable. The results for task 1 are shown in Table I and the case of a nominal MPC is explored for comparison purposes.
From Table I, the advantage of barrier MPC compared to the nominal MPC becomes obvious. The maximum gain for the case of barrier MPC is 2.913 compared to 1.130 for the nominal MPC. Additionally, after trial and error, we found that for $\kappa=3.4$, barrier MPC is destabilized, which is very close to the the computed value. Additionally, for $\kappa=2.9$, some simulations have been conducted for various $\mu$. From Fig. 5 (b) the advantage of the barrier MPC is clear, since the nominal MPC is unstable as expected since the maximum computed $\kappa$ is 1.130 .

TABLE I

MAXIMUM $K$ FOR STABILITY

\begin{tabular}{lcc}
\hline & Nominal & Barrier \\
\hline General & - & 1.091 \\
$\mathrm{ZF}\left(N_{Z F}=10\right)$ & - & 1.091 \\
$\mathrm{C}-Z \mathrm{ZF}\left(N_{Z F}=1\right)$ & 1.130 & $\mathbf{2 . 9 1 3}$ \\
$\mathrm{C}-\mathrm{ZF}\left(N_{Z F}=10\right)$ & 1.130 & $\mathbf{2 . 9 1 3}$ \\
\hline
\end{tabular}

For the next task, the results are depicted in Table II. The analysis shows that barrier MPC is more robust than the nominal MPC, for all the different methods applied. Additionally, C-ZF seems to produce the least conservative results, predicting the system is stable for all possible $r$ even for $N_{Z F}=1$. Next, the design parameter is fixed at $r=0.1$

TABLE II

MINIMUM $r$ FOR $b=0.25$ (A) AND MAXIMUM $b$ FOR $r=0.1$ (B)

\begin{tabular}{lrccc}
\hline & \multicolumn{2}{c}{$(A)$} & \multicolumn{2}{c}{$(B)$} \\
& $r($ for $b=0.25)$ & \multicolumn{2}{c}{$b($ for $r=0.1)$} \\
\hline Nominal & Barrier & Nominal & Barrier \\
\hline ZF $\left(N_{Z F}=10\right)$ & 3.994 & 1.150 & - & 0.0955 \\
C-ZF $\left(N_{Z F}=10\right)$ & 3.568 & 0.724 & - & 0.0986 \\
C-ZF $\left(N_{Z F}=20\right)$ & 0.098 & $\mathbf{0 . 0 0 0 1}$ & 0.2510 & $\mathbf{0 . 5 1 1 2}$ \\
\hline
\end{tabular}

and parameter $b$ of the uncertainty changes. The results are depicted in Table II (B). For this task, the robustness of the barrier MPC is again demonstrated, since most of the methods for nominal MPC failed to predict a stable system. However, all the stability tests performed for the barrier MPC predict a stable region. Conservatism can be reduced even further by including more IQCs with respect to the uncertainty. It should be noted that there is no constraint violation included in this example, however there is a theoretical guarantee for constraint violations when a quadratic function $\beta_{i}$ is employed [21].

\section{CONCLUSiON}

In this work input-to-output stability results are developed the case of barrier MPC. The barrier can improve the robustness of the MPC due to the change in the slope. Additionally, general convex constraints can be employed. Tighter timeinvariant convex constraints as well as staged constraints are considered. The tighter the constrained case the less conservative the analysis can become, through the use of dynamic multipliers. A convex search is presented that allows the 


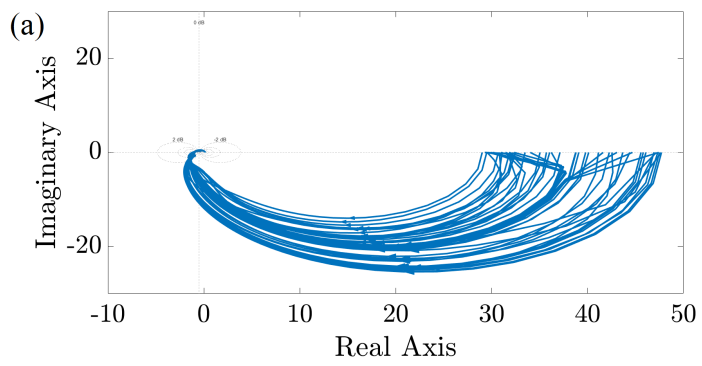

(b)

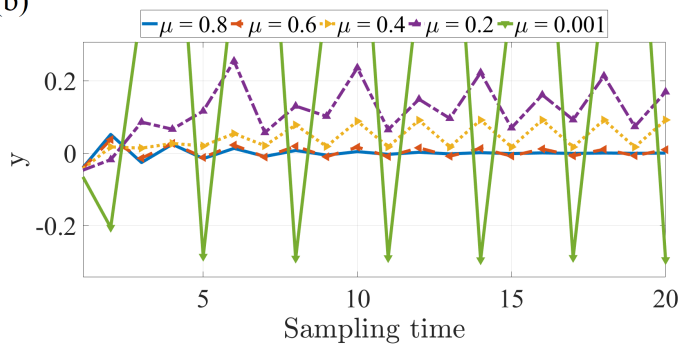

Fig. 5. (a) Nyquist diagram for the full plant of the numerical example. (b) Simulations for Task 1, where the output starts to oscillate for small $\mu$.

application of stability criteria using multipliers. An important benefit of the presented methodology is its applicability to nonlinear systems that can be represented as piecewise affine (PWA) systems extending our recent work [35].

All Appendices are available online at [36]

\section{ACKNOWLEDGMENT}

University of Manchester Presidential Doctoral Scholarship Award to PP is gratefully acknowledged.

\section{REFERENCES}

[1] D. Mayne, J. Rawlings, C. Rao, and P. Scokaert, "Constrained model predictive control: Stability and optimality," Automatica, vol. 36, no. 6, pp. 789-814, 2000.

[2] V. A. Yakubovich, "Frequency conditions for the absolute stability of control systems with several nonlinear or linear nonstationary blocks," Avtomat. i Telemekh., pp. 5-30, 1967.

[3] A. Megretski and A. Rantzer, "System analysis via integral quadratic constraints," IEEE Transactions on Automatic Control, vol. 42, no. 6, pp. 819-830, 1997.

[4] J. Veenman, C. W. Scherer, and H. Köroğlu, "Robust stability and performance analysis based on integral quadratic constraints," European Journal of Control, vol. 31, pp. 1-32, 2016.

[5] H. Pfifer and P. Seiler, "Integral quadratic constraints for delayed nonlinear and parameter-varying systems," Automatica, vol. 56, pp. 36 $-43,2015$.

[6] M. Cantoni, U. T. Jönsson, and C. Y. Kao, "IQC robustness analysis for feedback interconnections of unstable distributed parameter systems," Proceedings of the IEEE Conference on Decision and Control, pp. 11241130,2009

[7] U. Jönsson and A. Rantzer, Advances in Linear Matrix Inequality Methods in Control. Society for Industrial and Applied Mathematics, 2000, ch. Optimization of Integral Quadratic Constraints, pp. 109-127.

[8] W. P. Heath, G. Li, A. G. Wills, and B. Lennox, "The robustness of input constrained model predictive control to infinity-norm bound model uncertainty," IFAC Proceedings Volumes, vol. 39, no. 9, pp. 495-500, 2006.

[9] C. Løvaas, M. Seron, and G. Goodwin, "Robust output-feedback model predictive control for systems with unstructured uncertainty," Automatica, vol. 44, no. 8, pp. 1933-1943, 2008.
[10] P. Petsagkourakis, W. P. Heath, and C. Theodoropoulos, "Stability analysis of piecewise affine systems with multi-model predictive control," Automatica, vol. 111, p. 108539, 2020

[11] P. S. G. Cisneros and H. Werner, "A dissipativity formulation for stability analysis of nonlinear and parameter dependent mpc," in 2018 Annual American Control Conference (ACC), June 2018, pp. 3894-3899.

[12] G. Zames and P. L. Falb, "Stability conditions for systems with monotone and slope-restricted nonlinearities," SIAM Journal on Control, vol. 6, no. 1, pp. 89-108, 1968.

[13] W. P. Heath and A. G. Wills, "Zames-Falb multipliers for quadratic programming," IEEE Transactions on Automatic Control, vol. 52, no. 10, pp. 1948-1951, 2007.

[14] M. G. Safonov and V. V. Kulkarni, "Zames - Falb multipliers for MIMO nonlinearities," International Journal of Robust and Nonlinear Control, vol. 10 , no. $11-12$, pp. 1025-1038, 2000.

[15] F. D'Amato, M. Rotea, A. Megretski, and U. Jönsson, "New results for analysis of systems with repeated nonlinearities," Automatica, vol. 37, no. 5, pp. 739-747, 2001

[16] M. Fetzer and C. W. Scherer, "Absolute stability analysis of discrete time feedback interconnections," IFAC-PapersOnLine, vol. 50, no. 1, pp. 8447-8453, 2017.

[17] J. Carrasco, W. P. Heath, J. Zhang, N. S. Ahmad, and S. Wang, "Convex searches for discrete-time zames-falb multipliers," IEEE Transactions on Automatic Control, 2019, doi:10.1109/TAC.2019.2958848.

[18] A. G. Wills and W. P. Heath, "Barrier function based model predictive control," Automatica, vol. 40, no. 8, pp. 1415-1422, 2004.

[19] _ , "Application of barrier function based model predictive control to an edible oil refining process," Journal of Process Control, vol. 15, no. 2, pp. 183-200, 2005.

[20] Y. Wang and S. Boyd, "Fast Model Predictive Control Using Online Optimization," IEEE Transactions on Control Systems Technology, vol. 18 , no. 2, pp. 267-278, 2010

[21] C. Feller and C. Ebenbauer, "Relaxed logarithmic barrier function based model predictive control of linear systems," IEEE Transactions on Automatic Control, vol. 62, no. 3, pp. 1223-1238, 2016.

[22] _ "A stabilizing iteration scheme for model predictive control based on relaxed barrier functions," Automatica, vol. 80, pp. 328-339, 2017.

[23] K. Zhao and Y. Song, "Removing the feasibility conditions imposed on tracking control designs for state-constrained strict-feedback systems," IEEE Transactions on Automatic Control, vol. 64, no. 3, pp. 1265-1272, March 2019.

[24] Y. Song, Z. Shen, L. He, and X. Huang, "Neuroadaptive control of strict feedback systems with full-state constraints and unknown actuation characteristics: An inexpensive solution," IEEE Transactions on Cybernetics, vol. 48, no. 11, pp. 3126-3134, Nov 2018.

[25] C. Feller and C. Ebenbauer, "Input-to-state stability properties of relaxed barrier function based MPC," IFAC-PapersOnLine, vol. 48, no. 23, pp. 302-307, 2015

[26] J. Maciejowski, Predictive Control: With Constraints, ser. Pearson Education. Prentice Hall, 2002.

[27] R. Rockafellar, Convex Analysis, ser. Princeton landmarks in mathematics and physics. Princeton University Press, 1970.

[28] J. C. Willems, The Analysis of Feedback Systems, ser. Graham Foundation/Mit Press Series in Contemporary Architect. MIT Press, 1971.

[29] S. Boyd, S.-J. Kim, L. Vandenberghe, and A. Hassibi, "A tutorial on geometric programming," Optimization and Engineering, vol. 8, no. 1, p. 67, 2007.

[30] R. S. Varga, Matrix Iterative Analysis. Springer, 2000

[31] W. P. Heath and G. Li, "Multipliers for model predictive control with structured input constraints," Automatica, vol. 46, no. 3, pp. 562-568, 2010.

[32] J. Carrasco, W. P. Heath, and A. Lanzon, "Equivalence between classes of multipliers for slope-restricted nonlinearities," Automatica, vol. 49, no. 6 , pp. $1732-1740,2013$.

[33] A. Rantzer, "On the Kalman-Yakubovich-Popov Lemma for Positive Systems," IEEE Transactions on Automatic Control, vol. 61, no. 5, pp. $1346-1349,2016$.

[34] K. Zhou and J. Doyle, Essentials of Robust Control. Prentice Hall, 1998.

[35] P. Petsagkourakis, W. P. Heath, and C. Theodoropoulos, "Robust Stability Analysis for Barrier-Based Equation-free Multi-linear Model Predictive Control," Chemical Engineering Research and Design, vol. 144, pp. 237-246, 2018.

[36] P. Petsagkourakis, W. P. Heath, C. Joaquin, and C. Theodoropoulos, "Robust Stability of Barrier-Based Model Predictive Control: Supplementary Material," 2020. [Online]. Available: https://doi.org/10.5281/zenodo.3934668 